\title{
4-Nitroquinoline-1-Oxide Induces the Formation of Cellular Topoisomerase I-DNA Cleavage Complexes
}

\author{
Ze-Hong Miao, V. Ashutosh Rao, Keli Agama, Smitha Antony, \\ Kurt W. Kohn, and Yves Pommier \\ Laboratory of Molecular Pharmacology, Center for Cancer Research, National Cancer Institute, NIH, Bethesda, Maryland
}

\begin{abstract}
RecQ helicase BLM-deficient cells are characteristically hypersensitive to 4-nitroquinoline-1-oxide (4NQO). We recently reported that isogenic BLM-deficient cells (PNSG13) are more sensitive than BLM-complemented cells (PNSF5) to camptothecin, which specifically traps topoisomerase I cleavage complexes (Toplcc). We now report that PNSG13 are also 3.5-fold more sensitive to 4 NQO compared with PNSF5 and that 4 NQO induces higher levels of Toplcc and reduced histone $\gamma$-H2AX in PSNG13 than in PNSF5. Similarly, 4NQO induces more Toplcc in primary fibroblasts from a patient with Bloom syndrome than in normal human fibroblasts. 4NQO also induces Toplcc in colon cancer HCT116 and HT29 cells in a time- and concentration-dependent fashion. Of note, distinct from camptothecin, the Toplcc produced by $4 \mathrm{NQO}$ accumulate progressively after $4 \mathrm{NQO}$ addition and persist following $4 \mathrm{NQO}$ removal. The Toplcc induced by $4 \mathrm{NQO}$ are detectable by alkaline elution. To examine the functional relevance of the Toplcc induced by 4 NQO, we used two stable topoisomerase I small interfering RNA (siRNA) cell lines derived from HCT116 and MCF7 cells. Both topoisomerase I siRNA cell lines are resistant to $4 \mathrm{NQO}$, indicating that Toplcc contribute to the cellular activity of $4 \mathrm{NQO}$. Collectively, these data show that $4 \mathrm{NQO}$ is an effective inducer of cellular Toplcc. Because 4NQO does not directly trap Toplcc in biochemical assays, we propose that active metabolites of $4 \mathrm{NQO}$ trap Toplcc by forming DNA adducts. Induction of Toplcc and histone $\gamma$-H2AX by 4 NQO may contribute to the cellular effects of $4 \mathrm{NQO}$, including its selective activity toward RecQ helicase BLM-deficient cells. (Cancer Res 2006; 66(13): 6540-5)
\end{abstract}

\section{Introduction}

Nuclear DNA topoisomerase I is essential for relaxing DNA supercoiling generated during replication, transcription, repair, and recombination (1-4). Topoisomerase I-mediated DNA relaxation proceeds by inducing of single-strand breaks (SSB) that allows the broken DNA strand to rotate around the complementary intact strand and then by rapidly sealing the broken phosphate backbone to restore the DNA continuity. DNA cleavage is due to the formation of transient covalent topoisomerase I-DNA intermediates, which are called topoisomerase I cleavage complexes (Toplcc). Camptothecin and other anticancer topoisomerase I inhibitors, such as indolocarbazoles and indenoisoquinolines, trap Toplcc reversibly by

Requests for reprints: Yves Pommier, Laboratory of Molecular Pharmacology, Center for Cancer Research, National Cancer Institute, NIH, Building 37, Room 5068, Bethesda, MD 20892-4255. Phone: 301-496-5944; Fax: 301-402-0752; E-mail: pommier@ nih.gov.

(c)2006 American Association for Cancer Research.

doi:10.1158/0008-5472.CAN-05-4471 binding specifically at the interface of the topoisomerase I-DNA complexes $(5,6)$. Stabilized cleavage complexes are converted into irreversible DNA breaks (topoisomerase I covalent complexes), as replication or transcription complexes collide into the Toplcc. Exogenous and endogenous DNA lesions, such as 8-oxoguanosine, SSB, UV lesions, and carcinogenic adducts, can also trap Toplcc and thereby induce topoisomerase I-mediated DNA damage (7-11).

RecQ helicase-deficient cells (WRN and BLM in humans and Sgs1 in yeast) are hypersensitive to camptothecin (refs. 5, 12, 13; http://discover.nci.nih.gov/pommier/pommier.htm), and we recently found that BLM-deficient cells (PSNG13) are not only more sensitive to camptothecin but also form increased Toplcc in response to camptothecin compared with their BLMcomplemented counterparts (PSNF5; ref. 14). BLM cells are also characteristically hypersensitive to 4-nitroquinoline-1-oxide (4NQO; refs. 15-20) with possible defects in apoptosis (21), but the mechanisms of this hypersensitivity have thus far remained unexplained. Genetic deficiency for the BLM RecQ helicase results in Bloom syndrome, a rare autosomal recessive genetic disease characterized by high frequency of sister chromatid exchanges (22) and predisposition to a wide variety of cancers occurring at an early age (23). The BLM protein functions in association with topoisomerase III $\alpha$ as a helicase-topoisomerase complex that can decatenate and resolve stalled replication forks (23).

The carcinogenic and mutagenic properties of $4 \mathrm{NQO}$ were first reported in 1957 (24). Its metabolic activation pathways have since been elucidated. $4 \mathrm{NQO}$ is metabolized into 4-acetoxyaminoquinoline-1-oxide (Ac-4HAQO), which can form covalent adducts to $C^{8}$ or $N^{2}$ of deoxyguanine and $N^{6}$ of deoxyadenine in DNA $(25,26)$. 4 NQO also produces oxidative damage and DNA SSB $(25,27,28)$. In the current study, we show that $4 \mathrm{NQO}$ effectively induces the formation of irreversible Toplcc with a greater frequency in BLMdeficient cells. These Toplcc are readily detectable in 4NQO-treated cells as protein-associated DNA SSB (PASB), which are characteristic of Toplcc (29), but cannot be formed with recombinant topoisomerase I in biochemical assays. We propose that 4NQOinduced DNA adducts trap Toplcc irreversibly and that these Toplcc are involved in the cellular effects of $4 \mathrm{NQO}$, including the selective activity toward RecQ helicase BLM-deficient cells.

\section{Materials and Methods}

Drugs and enzymes. 4NQO, camptothecin, and etoposide (VP-16) were purchased from Sigma-Aldrich Co. (St. Louis, MO). Each of these drugs was prepared as $10 \mathrm{mmol} / \mathrm{L}$ stock solutions in $100 \%$ DMSO. Aliquots were stored at $-20^{\circ} \mathrm{C}$, thawed, and diluted with complete medium just before each experiment. The final concentration of DMSO in culture medium did not exceed $0.1 \%(\mathrm{v} / \mathrm{v})$. Recombinant human topoisomerase I was purified from TN5 insect cells (HighFive, Invitrogen Corp., San Diego, CA) using a baculovirus construct for the $\mathrm{NH}_{2}$-terminal truncated human topoisomerase I cDNA as described previously (30). Recombinant human topoisomerase II $\alpha$ was expressed in yeast and purified as described (31). 
Cell culture. Human colon carcinoma HCT116 and HT29 cell lines were provided by the National Cancer Institute Developmental Therapeutics program (Dr. Nick Scudiero). Topoisomerase I small interfering RNA (siRNA) cells HCT116-siRNA-Topl and MCF7-siRNA-Topl and their respective control cell lines, which were established with HCT116 and human breast cancer MCF7 cell lines by stable topoisomerase I siRNA transfection, have been described elsewhere (32). Normal human skin fibroblast GM00037 cells and skin fibroblasts GM01492 from a patient with Bloom syndrome were obtained from Coriell Cell Repositories (Camden, NJ). Cells were grown in DMEM supplemented with $10 \%$ (v/v) heat-inactivated FCS, 100 units $/ \mathrm{mL}$ penicillin, and $100 \mu \mathrm{g} / \mathrm{mL}$ streptomycin (Life Technologies, Grand Island, $\mathrm{NY})$ at $37^{\circ} \mathrm{C}$ in a $5 \% \mathrm{CO}_{2}$ humidified atmosphere. Isogenic BLM-deficient PSNG13 and BLM-complemented PSNF5 fibroblasts (33) were generously provided by Dr. Ian Hickson (Oxford, United Kingdom) and cultured under the same conditions as the above, except for the addition of G-418 (340 $\mu \mathrm{g} / \mathrm{mL}$; Sigma-Aldrich) in the culture medium.

Assessment of cytotoxicity. Cytotoxicity of $4 \mathrm{NQO}$ was assessed by the sulforhodamine B (Sigma-Aldrich) assay (34). $\mathrm{IC}_{50}$ was calculated using the software Prism 4 (GraphPad Software, Inc., San Diego, CA). Relative resistance, called resistance factor, was expressed as the ratio of the $\mathrm{IC}_{50}$ of resistant cells to $\mathrm{IC}_{50}$ of corresponding sensitive cells. Each experiment was done at least twice in triplicate.

Detection of topoisomerase I-DNA complexes. Covalent topoisomerase I-DNA cleavage complexes were detected by the immunocomplex of enzyme bioassay $(32,35)$. Briefly, $1 \times 10^{6}$ cells were lysed in $1 \%$ Sarkosyl and homogenized with a Dounce homogenizer. The cell lysates were gently layered on $\mathrm{CsCl}$ step gradients and centrifuged at $165,000 \times g$ at $20^{\circ} \mathrm{C}$ for 20 hours. Thirteen consecutive $0.5 \mathrm{~mL}$ fractions were collected, diluted with an equal volume of $25 \mathrm{mmol} / \mathrm{L}$ sodium phosphate $(\mathrm{pH} \mathrm{6.6)}$ ), and applied to Immobilon-P membranes (Millipore Corp., Billerica, MA) using a slotblot vacuum manifold. Topoisomerase I-DNA complexes were detected by immunoblotting using the C21 topoisomerase I monoclonal antibody (a kind gift from Dr. Yung-Chi Cheng, Yale University, New Haven, CT) or the topoisomerase II polyclonal antibody from the Abcam, Inc. (Cambridge, MA). All experiments were done independently at least twice.

Confocal microscopy of $\boldsymbol{\gamma}$-H2AX foci formation. Cells used for microscopic analysis were grown in Nunc chamber slides (Nalgene, Rochester, NY) using $0.5 \mathrm{~mL}$ growth medium. For $\gamma$-H2AX foci detection, cells were fixed and permeabilized as described previously using $4 \%$ paraformaldehyde and cold $70 \%$ ethanol (14). Nonspecific binding was blocked using $8 \%$ bovine serum albumin in PBS. Fixed cells were stained overnight with anti-mouse monoclonal primary antibodies (in $1 \%$ bovine serum albumin at $4^{\circ} \mathrm{C}$; Upstate Technology, Charlottesville, VA) and tagged with fluorescent secondary antibodies (Molecular Probes, Inc., Carlsbad, CA) for 2 hours at room temperature. Slides were mounted using Vectashield mounting liquid (Vector Laboratories, Burlingame, CA) and sealed. Prepared slides were visualized using a Nikon (Melville, NY) Eclipse TE-300 confocal laser scanning microscope system and stored as TIFF images.

Alkaline elution assays. Alkaline elution assays were used to quantitate DNA SSB and to estimate DNA-protein cross-links, from which the production of PASB was inferred (36-38). Cellular DNA was radiolabeled with $1 \mu \mathrm{Ci} / \mathrm{mL}\left[{ }^{3} \mathrm{H}\right]$ thymidine (Perkin-Elmer Life Science Co., Boston, MA) for 48 hours at $37^{\circ} \mathrm{C}$ and chased in nonradioactive medium overnight. After drug treatments, cells were scraped in HBSS and counted to determine the appropriate loading. Aliquots were placed in drug-containing ice-cold HBSS. After alkaline elution, filters were incubated at $65^{\circ} \mathrm{C}$ with $1 \mathrm{~mol} / \mathrm{L}$ $\mathrm{HCl}$ for 45 minutes and $0.04 \mathrm{~mol} / \mathrm{L} \mathrm{NaCl}$ was added for an additional 45 minutes. Radioactivity in all fractions was measured with a liquid scintillation analyzer (Packard Instruments, Meridien, CT). DNA-protein cross-links were analyzed under nondeproteinizing, DNA-denaturing conditions using protein-adsorbing filters $(29,36,38)$. SSB were assessed under deproteinizing, DNA-denaturing conditions using filters that do not adsorb proteins (polycarbonate filters; refs. 36, 38). SSB frequencies were calculated as $\left[\log \left(R_{\mathrm{t}} / R_{0}\right) / \log \left(R_{3} / R_{0}\right)\right] \times 300$, where $R_{\mathrm{t}}, R_{0}$, and $R_{3}$ correspond to the DNA selections for $4 \mathrm{NQO}$-treated cells, untreated cells, and cells treated with $3 \mathrm{~Gy}$, respectively.
Induction of topoisomerase I and II cleavage complexes in cell-free systems. To test whether $4 \mathrm{NQO}$ directly trapped topoisomerase I or II, recombinant topoisomerase I and II enzyme cleavage assays were done as described (9, 39). Imaging was done using a PhosphorImager (Molecular Dynamics, Sunnyvale, CA)

Western blot analyses. Topoisomerase I levels in topoisomerase I siRNA cell lines and the corresponding control cell lines and in the BLM-deficient and BLM-proficient cells were measured by Western blotting with the C21 topoisomerase I monoclonal antibody. Protein bands were quantified with the software from Li-Cor Bioscience (Lincoln, NE). The experiments were done at least twice independently, and data are expressed as mean \pm SD.

\section{Results}

Differential cytotoxicity, Toplcc, and $\gamma$-H2AX foci induced by $4 N Q O$ in BLM-deficient and BLM-proficient cells. We recently reported that BLM-deficient cells (PNSG13) are more sensitive to camptothecin than isogenic BLM-complemented cells (PNSF5; ref. 14). Figure $1 A$ shows that PNSG13 are also 3.5-fold more sensitive to $4 \mathrm{NQO}$ than PNSF5. The $\mathrm{IC}_{50}$ for PSNG13 cells was $49.4 \pm 4.7 \mathrm{nmol} / \mathrm{L}$, whereas the BLM-complemented cells exhibited an $\mathrm{IC}_{50}$ of $173.8 \pm 15 \mathrm{nmol} / \mathrm{L}$. To detect whether $4 \mathrm{NQO}$ can induce Toplcc, we used the immunocomplex of enzyme bioassay $(14,35)$. 4NQO produced Toplcc in both PNSG13 and PNSF5 (Fig. 1B). However, PNSG13 produced more Toplcc in response to 4NQO than PNSF5 (Fig. 1B). Toplcc were 2.62-fold higher in PNSG13 than in PNSF5 at $10 \mu \mathrm{mol} / \mathrm{L} 4 \mathrm{NQO}$ and 1.57 -fold higher at $30 \mu \mathrm{mol} / \mathrm{L}$ 4NQO (Fig. $1 B$ ). 4NQO also induced $\sim 2$-fold higher frequency of Toplcc in the untransformed, primary GM01492 fibroblasts from a patient with Bloom syndrome than in primary normal human GM00037 fibroblasts (Fig. 1C). To test whether the difference in topoisomerase I trapping could be attributed to different topoisomerase I expression levels, we assayed topoisomerase I protein levels using Western blotting of whole-cell extracts. The level of topoisomerase I protein in the BLM-deficient and BLM-proficient cells were comparable (Fig. $1 A$ ) and thus could not account for the greater Toplcc in the BLM-deficient cells. These results show that 4NQO induces Toplcc in all four cell lines examined and that BLM-deficient cells tend to form more Toplcc that are not due to differences in global topoisomerase I protein. We further examined the formation of $\gamma-\mathrm{H} 2 \mathrm{AX}$ foci, which are formed in response to Toplcc (14, 40), and found that treatment of PSNG13 (BLMdeficient) cells with 4NQO also produced reduced $\gamma$-H2AX compared with PSNF5 (BLM-proficient) cells (Fig. 1D) as shown previously with camptothecin in the same cell lines (14).

4NQO induces irreversible Toplcc in a time- and concentration-dependent manner. Using camptothecin as the positive control, we examined the features of Toplcc induction by 4NQO. In human colon carcinoma HCT116 cells, 4NQO also induced the formation of Toplcc in a time- and concentration-dependent fashion (Fig. 2A). Relatively few Toplcc were detectable after 30-minute exposure to $4 \mathrm{NQO}$ and Toplcc were readily detectable after 1 or 3 hours with levels comparable with $5 \mu \mathrm{mol} / \mathrm{L}$ camptothecin (Fig. 2A). One-hour exposures at various $4 \mathrm{NQO}$ concentrations showed that Toplcc were easily detectable at concentrations above $10 \mu \mathrm{mol} / \mathrm{L} 4 \mathrm{NQO}$ in two human colon carcinoma cells (HCT116 and HT29; Fig. $2 A$ and $B$ ).

Camptothecin-induced Toplcc are characterized by their reversibility on drug removal $(29,41)$. Accordingly, a 1-hour postincubation of both HT29 (Fig. 2B) and HCT116 (data not shown) cells after camptothecin removal drastically reduced the Toplcc. In striking contrast, the $4 \mathrm{NQO}$-induced Toplcc remained 
A

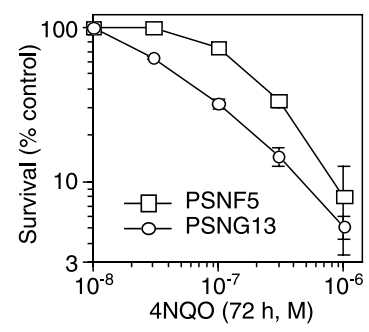

B

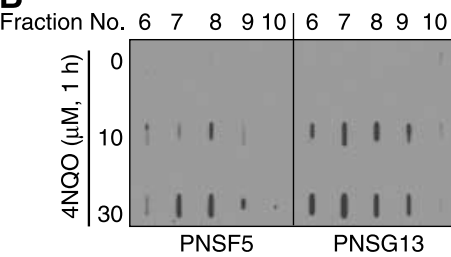

C

Fraction No. \begin{tabular}{lllll|lllll}
6 & 7 & 8 & 9 & 10 & 6 & 7 & 8 & 9 & 10
\end{tabular}

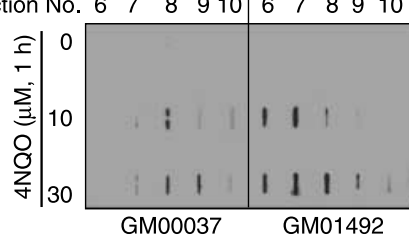

D

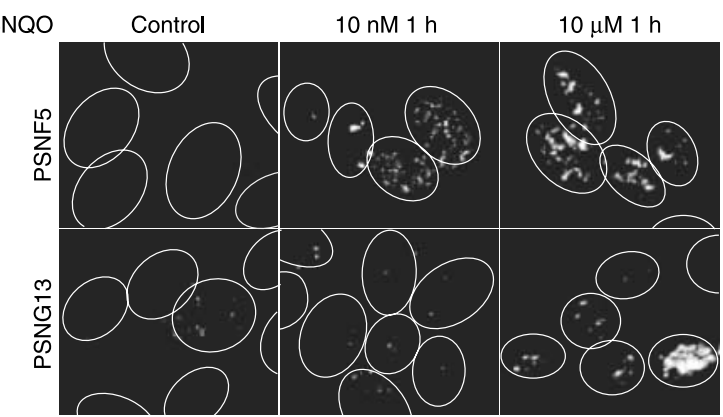

Figure 1. Differential sensitivity, induction of topoisomerase I-DNA complexes, and $\gamma-\mathrm{H} 2 \mathrm{AX}$ foci by $4 \mathrm{NQO}$ in BLM-deficient and BLM-proficient cells. $A$, survival curves of BLM-deficient PNSG13 and BLM-complemented PNSF5 cells exposed to $4 \mathrm{NQO}$ for 72 hours were determined by the sulforhodamine B assay. Experiments were conducted twice in triplicate independently. Points, mean; bars, SD. Basal levels of topoisomerase I protein in BLM-deficient (PSNG13 and GM01492) and BLM-proficient cells (BLM-complemented PSNF5 and normal GM0037 fibroblasts) were detected by Western blotting. $B$, induction of topoisomerase I-DNA complexes by 4NQO in BLM-deficient (PSNG13) and BLM-complemented (PSNF5) cells detected by the immunocomplex of enzyme bioassay. Quantitation of the results was conducted with PhotoShop 7.0. Values were calculated as (total pixel intensity of the five bands corresponding to fractions $6-10)$ - (total pixel intensity of the corresponding bands in the absence of $4 \mathrm{NQO}$ ). $C$, as in (B) for the primary Bloom syndrome fibroblasts (GM01492) and normal fibroblasts (GM00037). D, reduced formation of $\gamma-\mathrm{H} 2 \mathrm{AX}$ foci in BLM-deficient cells treated with 4NQO. Exponentially growing PSNG13 (BLM-deficient) or PSNF5 (BLM-complemented) cells were exposed to 10 or $10 \mathrm{mmol} / \mathrm{L} 4 \mathrm{NQO}$ for 1 hour. After slide preparation as described in Materials and Methods, cells were studied for formation of microscopic nuclear foci corresponding to phosphorylated histone $\mathrm{H} 2 \mathrm{AX}, \gamma-\mathrm{H} 2 \mathrm{AX}$. Color images were converted to grayscale, and nuclear outlines were superimposed using corresponding propidium iodide images. Representative images from independent experiments.

stable even for 3-hour incubation following 4NQO removal (Fig. 2B), indicating the irreversibility of the Toplcc induced by 4 NQO.

Additionally, 4NQO did not produce any detectable covalent Top2cc in HCT116 or HT29 under conditions where Toplcc

were readily detected (Fig. $2 C$ ). These experiments show that 4NQO induces irreversible Toplcc but is ineffective against topoisomerase II.

4NQO generates PASB in cells. Protein-linked DNA breaks are characteristic of topoisomerase cleavage complexes $(3,36)$. DNA SSB mediated by topoisomerases can be detected by alkaline elution only under deproteinizing conditions $(29,42)$ as each break is associated with a topoisomerase molecule (38). This is in contrast with frank breaks, such as those induced by ionizing radiation that can be similarly detected without or with deproteination (36). We used the alkaline elution to detect whether 4NQO induced SSB and whether these breaks corresponded to PASB (29). Alkaline elution is a sensitive and quantitative method for the detection of SSB (38). 4NQO induced high SSB frequency ( $\sim 900 \mathrm{rad}$ equivalent SSB $)$ at 3 $\mu \mathrm{mol} / \mathrm{L}$ in HCT116 (Fig. 3). Deproteinizing conditions were required to detect these breaks, which was not the case for 3 Gy (Fig. $3 A$ ). These results are consistent with the induction by 4 NQO of PASB in the form of Toplcc.

Exposure of HCT116 cells to increasing concentrations of $4 \mathrm{NQO}$ for 1 hour and quantitation of independent alkaline elution experiments showed that $4 \mathrm{NQO}$ induces increasing SSB at

\section{A}

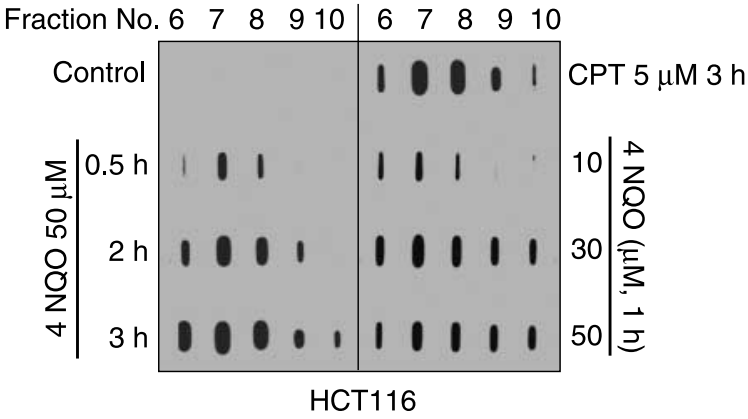

B

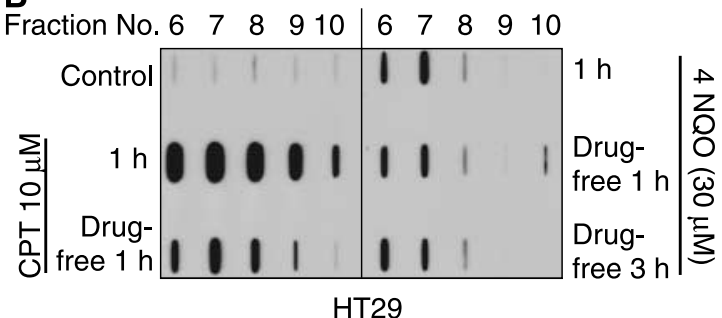

C

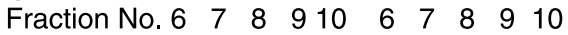

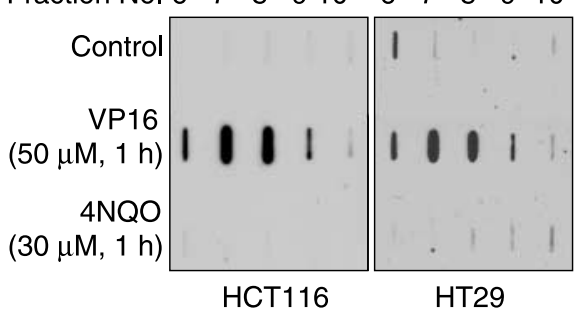

Figure 2. $4 \mathrm{NQO}$ produces irreversible Top1cc but no Top2cc. $A$, time course and concentration dependence of Top1cc induction by 4NQO in HCT116 cells. Camptothecin was used as a positive control. $B$, persistence of the Top1cc induced by 4NQO in HT29 cells on 4NQO removal for 1 or 3 hours. Note the reversibility of camptothecin-induced Top1cc. C, 4NQO does not produce Top2cc. VP-16 was used as a positive control for Top2cc formation. 


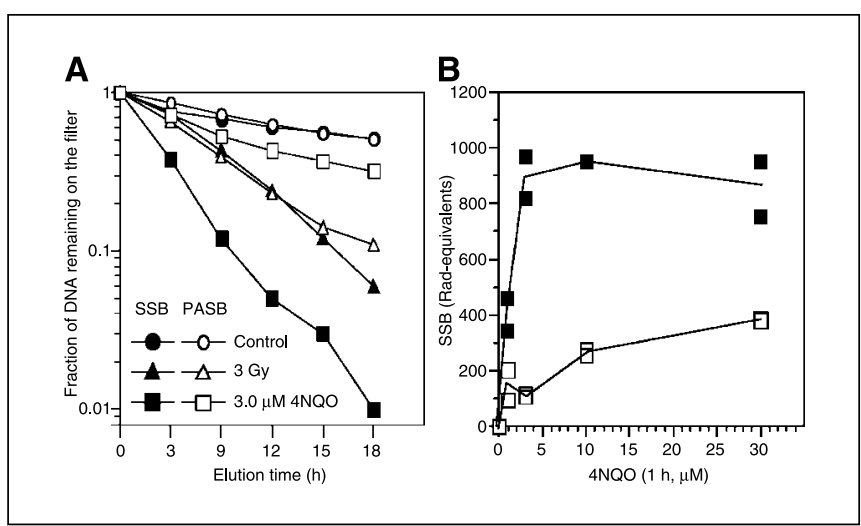

Figure 3. $4 \mathrm{NQO}$ induces PASB. SSB and non-PASB produced in HCT116 cells treated with $4 \mathrm{NQO}$ for 1 hour were detected by the alkaline elution assay. $A$, representative alkaline elution experiment showing total SSB (measured under deproteinizing conditions; closed symbols) and non-PASB (measured under nondeproteinizing conditions; open symbols) in cells exposed to $3 \mu \mathrm{mol} / \mathrm{L}$ $4 \mathrm{NQO}$ for 1 hour. $B$, dose response for SSB (closed symbols) and non-PASB (open symbols) induced by 4NQO. Alkaline elution results are expressed in rad equivalents as described in Materials and Methods. Data at each concentration of $4 \mathrm{NQO}$ represent two independent determinations.

concentration below $3 \mu \mathrm{mol} / \mathrm{L}$ and that SSB reach a plateau at and above $3 \mu \mathrm{mol} / \mathrm{L}$ (Fig. $3 B$ ). As the $4 \mathrm{NQO}$ concentration increased, 4NQO induced detectable SSB under nondeproteinizing conditions, but this signal was much lower than the SSB signal under deproteinizing conditions (Fig. $3 B$, open symbols). This result is consistent with the production of PASB by $4 \mathrm{NQO}$ together with a smaller component of protein-free SSB at high concentrations.

4NQO does not directly inhibit topoisomerase I or II enzymes. To test whether induction of cellular Toplcc by $4 \mathrm{NQO}$ is due to a direct effect on topoisomerase I enzyme, we did biochemical analyses with recombinant human topoisomerase I enzyme. In this system, camptothecin was used as a positive control to show the direct trapping of Toplcc (Fig. 4A, lane 3). However, 4NQO, even at a high concentration of $100 \mu \mathrm{mol} / \mathrm{L}$, produced a comparable profile of bands as topoisomerase I enzyme alone, which shows lack of direct topoisomerase I inhibition by 4NQO (Fig. 4A). Similarly, no cleavage was observed for Top2cc under conditions where VP-16 produced Top2cc. These experiments show that $4 \mathrm{NQO}$ does not act as a direct topoisomerase inhibitor (Fig. 4B).

Topoisomerase I-deficient cells are resistant to 4NQO. To examine the functional relevance of the Toplcc induced by $4 \mathrm{NQO}$, we used two stable topoisomerase I siRNA cell lines derived from HCT116 and from breast cancer MCF7 cells. Both HCT116-siTop1 and MCF7-siTop1 cell lines express $\sim 20 \%$ topoisomerase I protein compared with their respective control cell lines (Fig. $5 A$, insets). Both HCT116-siTop1 and MCF7-siTop1 cell lines displayed 2-fold resistance to $4 \mathrm{NQO}$ compared with their corresponding control siRNA cell lines (Fig. 5A). We further compared the ability of 4NQO to form $\gamma$-H2AX foci in MCF-7-siTop1 and MCF-7-siRNA control cells. Figure $5 B$ and $C$ revealed that although both cell lines produced $\gamma-\mathrm{H} 2 \mathrm{AX}$ foci kinetically in response to $4 \mathrm{NQO}$ treatment the net increase of $\gamma$-H2AX foci in the topoisomerase I siRNA cells was significantly less than that in the corresponding control siRNA cells. These results show that the decreasing topoisomerase I protein level rescues the cells from the cell killing and DNA damage by 4 NQO, which indicates that Toplcc contributes to the cellular toxicity of $4 \mathrm{NQO}$.

\section{Discussion}

Toplcc result from the covalent binding of the catalytic tyrosyl group (human $\mathrm{Tyr}^{723}$ ) of the enzyme to the phosphate group at the 3 -terminus of the cleaved DNA strand at the moment and site that topoisomerase I enzyme attacks and breaks one strand of duplex DNA $(3,5,43)$. The covalent complexes reverse as the enzyme religates the broken DNA. Thus, Toplcc are the key intermediates by which topoisomerase I exerts its key DNA cleavage and religation activities. Under physiologic conditions, the existence of such complexes is fundamental to the basic function of topoisomerase I. As they are very transient, Toplcc are practically undetectable under physiologic conditions. Many exogenous and endogenous factors can reversibly or irreversibly trap topoisomerase I complexes and convert them into lethal intermediates as they lead to persistent DNA breaks and covalent topoisomerase I-DNA adducts (refs. 5, 12; http://discover.nci.nih.gov/pommier/pommier.htm).

In the current study, we report for the first time that the potent carcinogen and mutagen $4 \mathrm{NQO}$ effectively produces irreversible Toplcc. 4NQO elicits time- and concentration-dependent Toplcc that persist for $>3$ hours following $4 \mathrm{NQO}$ removal (Figs. 1 and 2). Alkaline elution assays further indicated the presence of cellular Toplcc as PASB, which are characteristic of topoisomerase
A
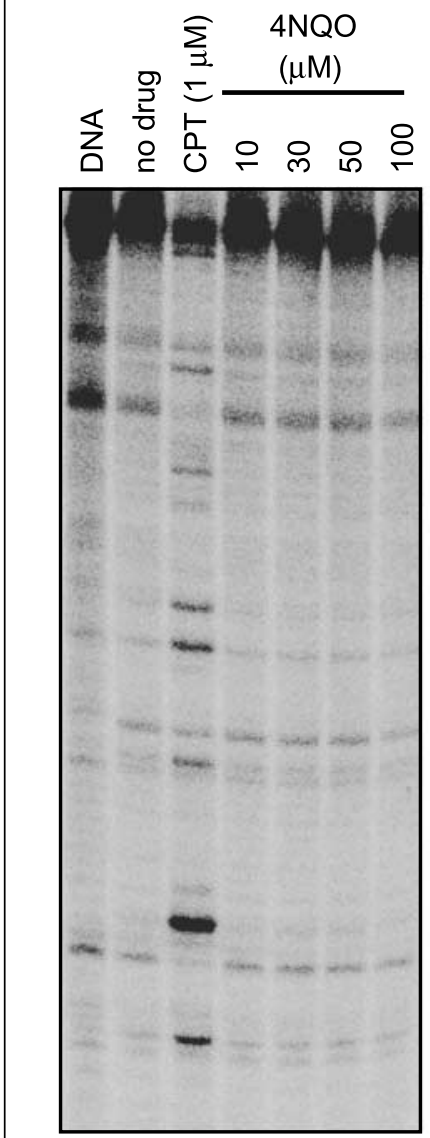

B

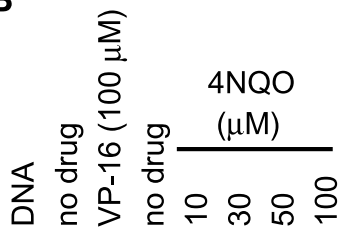

Figure 4. $4 \mathrm{NQO}$ does not produce topoisomerase I $(A)$ or topoisomerase II $(B)$ cleavage complexes in a cell-free system. Reactions were done at the indicated $4 \mathrm{NQO}$ for 20 minutes at $30^{\circ} \mathrm{C}$. Camptothecin $(C P T)$ and VP-16 were used as positive control for topoisomerase I and II, respectively. 


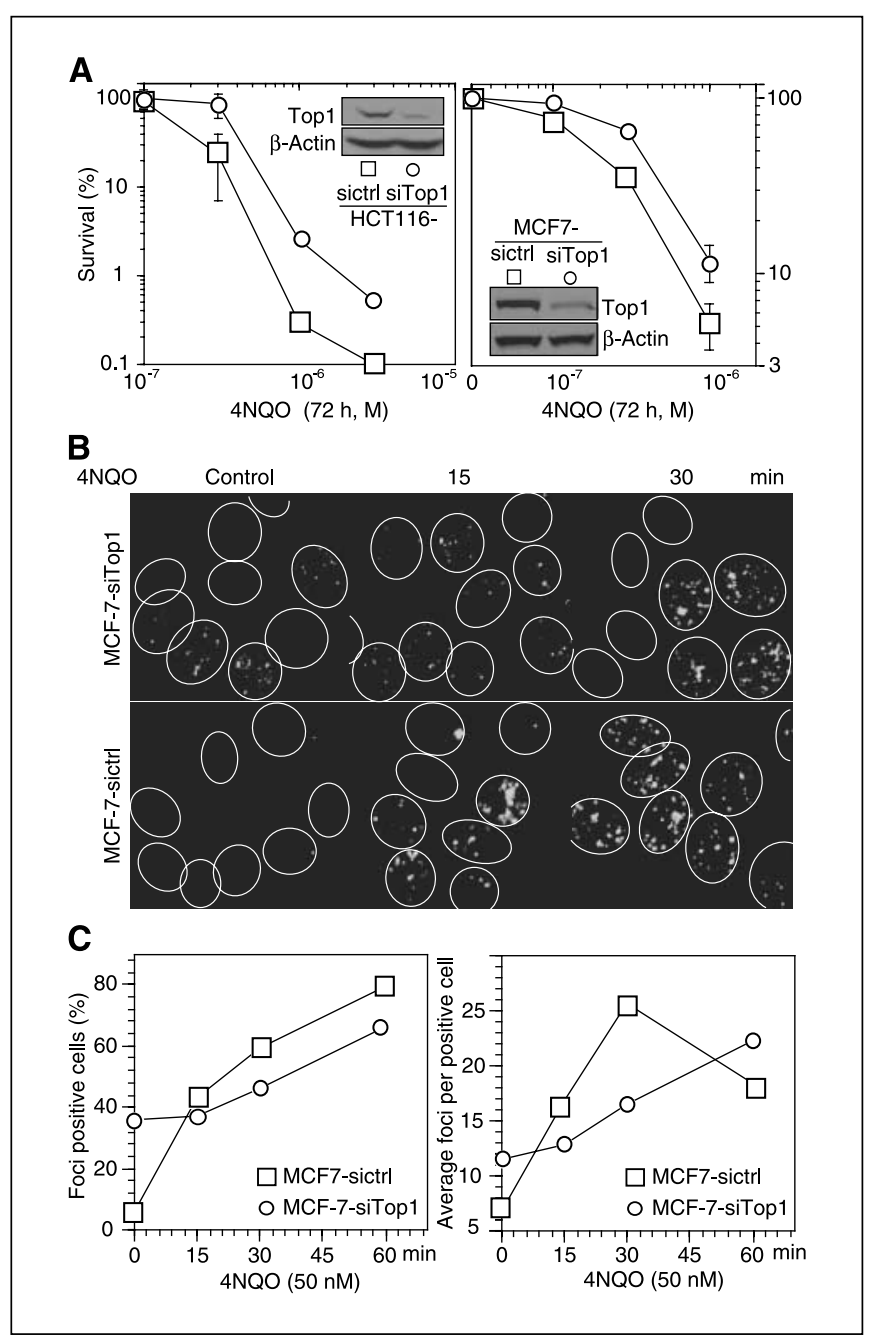

Figure 5. Reduction of topoisomerase I expression leads to partial resistance to $4 \mathrm{NQO}$ and reduced formation of $\gamma-\mathrm{H} 2 \mathrm{AX}$ foci by $4 \mathrm{NQO}$. $A$, survival curves of HCT116-siTop1 MCF7-siTop1 and their respective control siRNA cells. The cytotoxicity of $4 \mathrm{NQO}$ in two pairs of topoisomerase I siRNA tumor cell lines was determined by the sulforhodamine B assay. Insets, topoisomerase I expression in the topoisomerase I siRNA cell lines and their control cell was determined by Western blotting with $\beta$-actin as loading control. $B$, reduced formation of $\gamma-\mathrm{H} 2 \mathrm{AX}$ foci in MCF-7-siTop1 cells treated with 4NQO. Cells were exposed to $50 \mathrm{nmol} / \mathrm{L}$ 4NQO for 15, 30, and 60 minutes. After slide preparation as described in Materials and Methods, cells were studied for formation of microscopic nuclear foci corresponding to phosphorylated histone, $\gamma-\mathrm{H} 2 \mathrm{AX}$. Color images were converted to grayscale, and nuclear outlines were superimposed using corresponding propidium iodide images. Representative experiments. C, percentage of $\gamma-\mathrm{H} 2 \mathrm{AX}$ foci-positive cells and average foci per $\gamma-\mathrm{H} 2 \mathrm{AX}$-positive cell. At least 100 cells for each experimental group were counted.

cleavage complexes $(29,36)$. Increasing exposure to $4 \mathrm{NQO}$ also produced some frank breaks (Fig. 3). Collectively, our data show that $4 \mathrm{NQO}$ is an effective inducer of Toplcc in all human cell lines examined: in the four transformed cell lines [PSNG13, PSN15 (Fig. 1), HCT116, and HT29 (Figs. 2 and 3)] and in the two primary fibroblasts [GM00037 and GM01492 (Fig. 1)]. In contrast, 4NQO does not seem to affect topoisomerase II (Fig. $2 C$ ).

The induction of irreversible Toplcc by 4 NQO (Fig. 2) is not the consequence of a direct action of $4 \mathrm{NQO}$ on topoisomerase I as topoisomerase I cleavage activity is unaffected by $4 \mathrm{NQO}$ in cell-free system (Fig. 4). 4NQO is carcinogenic only after being metabolically activated (including via 4NQO reductase, EC1.7.1.9 in cytosol) to its ultimate carcinogen Ac-4HAQO (Fig. 6; refs. 25, 26, 44). Two active metabolites of Ac-4HAQO form three main carcinogenic DNA monoadducts: two on guanine (dG- $C^{8}$-AQO and dG- $\left.N^{2}-\mathrm{AQO}\right)$ and one on adenine (dA- $N^{6}-\mathrm{AQO}$; Fig. 6; ref. 25). It is well established that Toplcc can be trapped or induced by a wide variety of carcinogenic adducts at guanine $N^{2}$ [benzo[ $a$ ]pyrene (30), benzo[ $[c]$ phenanthrene (30), and acetaldehyde adducts (9)], guanine $O^{6}$ [methyl adducts (45)], and adenine $N^{6}$ [ethyl adducts (46); reviewed in ref. 7 and updated at http://discover.nci.nih.gov/ pommier/pommier.htm]. It is therefore plausible that the DNA monoadducts produced by $4 \mathrm{NQO}$ also elicit Toplcc. Because there were no necessary enzymes and other suitable physiologic conditions for $4 \mathrm{NQO}$ transformation in our cell-free system, 4NQO itself did not trap Toplcc as camptothecin did (Fig. 4).

Previous studies have indicated that SSB are induced in vitro and in vivo by 4NQO (47-50). Our experiments suggest that these breaks are mainly PASB, which probably correspond to Toplcc (Fig. 3). We found that $4 \mathrm{NQO}$ also induces "frank breaks" [i.e., non-protein-linked SSB that are detectable under nondeproteinizing conditions (Fig. 3); ref. 36]. Such frank breaks are less frequent than the PASB and increase with 4NQO concentration (Fig. 3). Whether such frank breaks contribute to the formation of Toplcc (51) or are a consequence of the irreversible Toplcc or other DNA lesions remains to be determined.

The finding of induction of Toplcc by 4NQO sheds some light on the cellular and biological effects of 4NQO. These Toplcc must contribute to the cellular damage and lethality resulting from 4NQO exposure as shown by the 2-fold resistance of cell lines with topoisomerase I down-regulation (Fig. 5) and by the greater sensitivity and increased Toplcc in BLM-deficient cells (Fig. 1). Thus, the formation of greater Toplcc in BLM-deficient cells provides a rationale for the hypersensitivity of Bloom syndrome

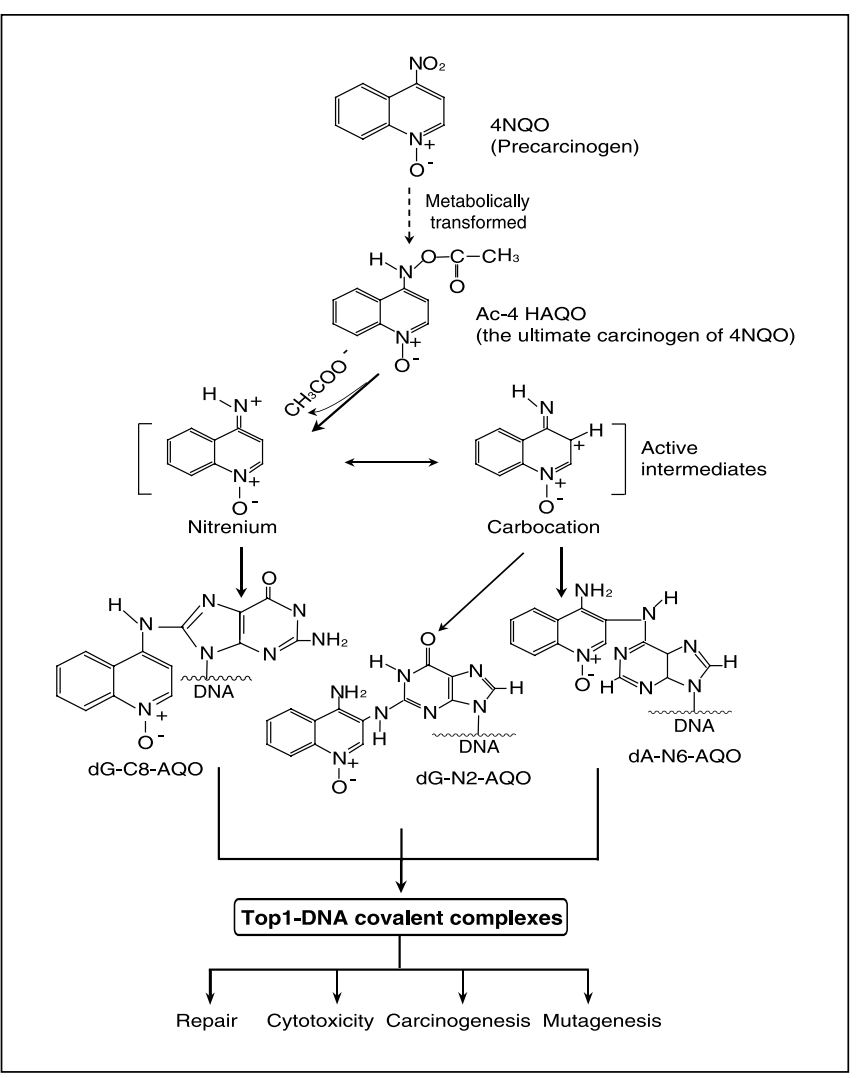

Figure 6. Schematic representation for the proposed mechanism and consequence of the $4 \mathrm{NQO}$-induced formation of covalent Top1cc. 
cells to 4NQO (Fig. 1). The finding that 4NQO generates Toplcc also offers a novel perspective on its molecular mechanisms of cytotoxicity, carcinogenicity, and mutagenicity. As 4NQO-induced DNA damage is an early event in its carcinogenesis $(52,53)$ and irreversible Toplcc induce recombinations (54), the covalent Toplcc produced by $4 \mathrm{NQO}$ may play a role in $4 \mathrm{NQO}$ carcinogenicity.

\section{Acknowledgments}

Received 12/15/2005; revised 3/28/2006; accepted 4/24/2006

Grant support: Intramural Research Program of the NIH, National Cancer Institute, Center for Cancer Research.

The costs of publication of this article were defrayed in part by the payment of page charges. This article must therefore be hereby marked advertisement in accordance with 18 U.S.C. Section 1734 solely to indicate this fact.

\section{References}

1. Leppard JB, Champoux JJ. Human DNA topoisomerase I: relaxation, roles, and damage control. Chromosoma 2005;114:75-85.

2. Nitiss JL. Investigating the biological functions of DNA topoisomerases in eukaryotic cells. Biochim Biophys Acta 1998;1400:63-81.

3. Wang JC. Cellular roles of DNA topoisomerases: a molecular perspective. Nat Rev Mol Cell Biol 2002;3: 430-40.

4. Pommier Y, Pourquier P, Fan Y, Strumberg D. Mechanism of action of eukaryotic DNA topoisomerase I and drugs targeted to the enzyme. Biochim Biophys Acta 1998;1400:83-105.

5. Pommier Y, Redon C, Rao VA, et al. Repair of and checkpoint response to topoisomerase I-mediated DNA damage. Mutat Res 2003;532:173-203.

6. Pommier Y, Marchand C. Interfacial inhibitors of protein-nucleic acid interactions. Curr Med Chem AntiCanc Agents 2005;5:421-9.

7. Pourquier P, Pommier Y. Topoisomerase I-mediated DNA damage. Adv Cancer Res 2001;80:189-216.

8. Lesher DT, Pommier Y, Stewart L, Redinbo MR. 8Oxoguanine rearranges the active site of human topoisomerase I. Proc Natl Acad Sci U S A 2002;99: 12102-7.

9. Antony S, Theruvathu JA, Brooks PJ, Lesher DT, Redinbo M, Pommier Y. Enhancement of camptothecin-induced topoisomerase I cleavage complexes by the acetaldehyde adduct $N^{2}$-ethyl-2'-deoxyguanosine. Nucleic Acids Res 2004;32:5685-92.

10. Pommier Y, Kohlhagen G, Pourquier P, Sayer JM, Kroth H, Jerina DM. Benzo[ $a$ ]pyrene epoxide adducts in DNA are potent inhibitors of a normal topoisomerase I cleavage site and powerful inducers of other topoisomerase I cleavages. Proc Natl Acad Sci U S A 2000; 97:2040-5.

11. Pommier Y, Laco GS, Kohlhagen G, Sayer JM, Kroth $\mathrm{H}$, Jerina DM. Position-specific trapping of topoisomerase I-DNA cleavage complexes by intercalated benzo[$a$ ]pyrene diol epoxide adducts at the 6-amino group of adenine. Proc Natl Acad Sci U S A 2000;97:10739-44.

12. Pommier Y, Barcelo J, Rao VA, et al. Repair of topoisomerase I-mediated DNA damage. Prog Nucleic Acid Res Mol Biol 2006;81:175-221.

13. Lee JW, Harrigan J, Opresko PL, Bohr VA. Pathways and functions of the Werner syndrome protein. Mech Ageing Dev 2005;126:79-86.

14. Rao VA, Fan AM, Meng L, et al. Phosphorylation of BLM, dissociation from topoisomerase III $\alpha$, and colocalization with $\gamma$-H2AX after topoisomerase I-induced replication damage. Mol Cell Biol 2005;25:8925-37.

15. Shiraishi Y. Bloom syndrome B-lymphoblastoid cells are hypersensitive towards carcinogen and tumor promoter-induced chromosomal alterations and growth in agar. EMBO J 1985;4:2553-60.

16. Shiraishi Y, Yosida TH, Sandberg AA. Malignant transformation of Bloom syndrome B-lymphoblastoid cell lines by carcinogens. Proc Natl Acad Sci U S A 1985; 82:5102-6.

17. Imamura O, Fujita K, Itoh $C$, Takeda S, Furuichi Y, Matsumoto T. Werner and Bloom helicases are involved in DNA repair in a complementary fashion. Oncogene 2002;21:954-63.

18. Poot M, Gollahon KA, Emond MJ, Silber JR, Rabinovitch PS. Werner syndrome diploid fibroblasts are sensitive to 4-nitroquinoline- $N$-oxide and 8-methoxypsoralen: implications for the disease phenotype. FASEB J 2002;16:757-8

19. Imamura O, Fujita K, Shimamoto A, et al. Bloom helicase is involved in DNA surveillance in early S phase in vertebrate cells. Oncogene 2001;20:1143-51.

20. Honma M, Tadokoro S, Sakamoto H, et al. Chromosomal instability in B-lymphoblastoid cell lines from Werner and Bloom syndrome patients. Mutat Res 2002; 520:15-24.

21. Wang $X W$, Tseng A, Ellis NA, et al. Functiona interaction of p53 and BLM DNA helicase in apoptosis. J Biol Chem 2001;276:32948-55.

22. German J. Bloom syndrome: a Mendelian prototype of somatic mutational disease. Medicine (Baltimore) 1993;72:393-406.

23. Hickson ID. RecQ helicases: caretakers of the genome. Nat Rev Cancer 2003;3:169-78.

24. Nakahara W, Fukuoka F, Sugimura T. Carcinogenic action of 4-nitroquinoline- $N$-oxide. Gann 1957;48:129-37. 25. Bailleul B, Daubersies $P$, Galiegue-Zouitina $S$, Loucheux-Lefebvre MH. Molecular basis of 4-nitroquinoline 1-oxide carcinogenesis. Jpn J Cancer Res 1989;80: 691-7.

26. Nagao M, Sugimura T. Molecular biology of the carcinogen, 4-nitroquinoline 1-oxide. Adv Cancer Res 1976;23:131-69.

27. Gebhart E, Bauer R, Raub U, Schinzel M, Ruprech KW, Jonas JB. Spontaneous and induced chromosomal instability in Werner syndrome. Hum Genet 1988;80: 135-9.

28. Baohong W, Jiliang H, Lifen J, et al. Studying the synergistic damage effects induced by $1.8 \mathrm{GHz}$ radiofrequency field radiation (RFR) with four chemical mutagens on human lymphocyte DNA using comet assay in vitro. Mutat Res 2005;578:149-57.

29. Covey JM, Jaxel C, Kohn KW, Pommier Y. Proteinlinked DNA strand breaks induced in mammalian cells by camptothecin, an inhibitor of topoisomerase I. Cancer Res 1989;49:5016-22.

30. Pommier Y, Kohlhagen G, Laco GS, Kroth H, Sayer JM, Jerina DM. Different effects on human topoisomerase I by minor groove and intercalated deoxyguanosine adducts derived from two polycyclic aromatic hydrocarbon diol epoxides at or near a normal cleavage site. J Biol Chem 2002;277:13666-72.

31. Wasserman RA, Austin CA, Fisher LM, Wang JC. Use of yeast in the study of anticancer drugs targeting DNA topoisomerases: expression of a functional recombinant human DNA topoisomerase II $\alpha$ in yeast. Cancer Res 1993:53:3591-6.

32. Sordet $\mathrm{O}$, Liao Z, Liu H, et al. Topoisomerase I-DNA complexes contribute to arsenic trioxide-induced apoptosis. J Biol Chem 2004;279:33968-75.

33. Gaymes TJ, North PS, Brady N, Hickson ID, Mufti GJ, Rassool FV. Increased error-prone non homologous DNA end-joining-a proposed mechanism of chromosomal instability in Bloom's syndrome. Oncogene 2002; 21:2525-33

34. Xiao D, Tan W, Li M, Ding J. Antiangiogenic potentia of 10-hydroxycamptothecin. Life Sci 2001;69:1619-28.

35. Subramanian D, Kraut E, Staubus A, Young DC, Muller MT. Analysis of topoisomerase I/DNA complexes in patients administered topotecan. Cancer Res 1995;55: 2097-103.

36. Kohn KW. DNA filter elution: a window on DNA damage in mammalian cells. BioEssays 1996;18:505-13. 37. Meng LH, Kohlhagen G, Liao ZY, Antony S, Sausville E, Pommier Y. DNA-protein cross-links and replicationdependent histone H2AX phosphorylation induced by aminoflavone (NSC 686288), a novel anticancer agen active against human breast cancer cells. Cancer Res 2005;65:5337-43.

38. Kohn KW. Principles and practice of DNA filter elution. Pharmacol Ther 1991;49:55-77.
39. Khan QA, Kohlhagen G, Marshall R, et al. Positionspecific trapping of topoisomerase II by benzo $[a]$ pyrene diol epoxide adducts: implications for interactions with intercalating anticancer agents. Proc Natl Acad Sci U S A 2003;100:12498-503.

40. Furuta T, Takemura H, Liao ZY, et al. Phosphorylation of histone $\mathrm{H} 2 \mathrm{AX}$ and activation of Mrell, Rad50, and Nbsl in response to replication-dependent DNA double-strand breaks induced by mammalian DNA topoisomerase I cleavage complexes. J Biol Chem 2003, 278:20303-12.

41. Hsiang YH, Liu LF. Identification of mammalian DNA topoisomerase I as an intracellular target of the anticancer drug camptothecin. Cancer Res 1988;48: 1722-6.

42. Ross WE, Glaubiger DL, Kohn KW. Protein-associated DNA breaks in cells treated with Adriamycin or ellipticine. Biochim Biophys Acta 1978;519:23-30.

43. Champoux JJ. DNA topoisomerases: structure, function, and mechanism. Annu Rev Biochem 2001;70: 369-413.

44. Galiegue-Zouitina S, Daubersies $P$, LoucheuxLefebvre MH, Bailleul B. Mutagenicity of $N^{2}$ guanylarylation is SOS functions dependent and reminiscent of the high mutagenic property of $4 \mathrm{NQO}$. Carcinogenesis 1989;10:1961-6.

45. Pourquier P, Waltman JL, Urasaki $Y$, et al. Topoisomerase I-mediated cytotoxicity of $N$-methyl- $N^{\prime}$ nitro- $N$-nitrosoguanidine: trapping of topoisomerase by the $O^{6}$-methylguanine. Cancer Res 2001;61:53-8.

46. Pourquier P, Bjornsti M-A, Pommier Y. Induction of topoisomerase I cleavage complexes by the vinyl chloride adduct, $1, N^{6}$-ethenoadenine. J Biol Chem 1998;273:27245-9.

47. Seo YR, Lee SH, Han SS, Ryu JC. Effect of p53 tumor suppressor on nucleotide excision repair in human colon carcinoma cells treated with 4-nitroquinoline 1oxide. Res Commun Mol Pathol Pharmacol 1999;104: 157-64.

48. Furihata C, Ikui E, Matsushima T. DNA single-strand scission in the pyloric mucosa of rat stomach induced by four glandular stomach carcinogens and three other chemicals. Mutat Res 1996;368:1-6.

49. Hasspieler BM, Ali FN, Alipour M, Haffner GD, Adel K. Human bioassays to assess environmental genotoxicity: development of a DNA break bioassay in HepG2 cells. Clin Biochem 1995;28:113-6.

50. Ohsawa K, Furihata C, Mori M, Ikui E. Ability of $N$-methyl- $N^{\prime}$-nitro- $N$-nitrosoguanidine, 4-nitroquinoline 1-oxide, dimethylnitrosamine, and $\mathrm{NaCl}$ to induce unscheduled DNA synthesis, stimulate replicative DNA synthesis, and produce DNA single-strand breaks in pyloric mucosa of rat stomach. Mutat Res 1993;287:307-19. 51. Pourquier P, Pilon AA, Kohlhagen G, Mazumder A, Sharma A, Pommier Y. Trapping of mammalian topoisomerase I and recombinations induced by damaged DNA containing nicks or gaps. Importance of DNA end phosphorylation and camptothecin effects. J Biol Chem 1997;272:26441-7.

52. Ribeiro DA, Favero Salvadori DM, da Silva RN Ribeiro Darros B, Alencar Marques ME. Genomic instability in non-neoplastic oral mucosa cells can predict risk during 4-nitroquinoline 1-oxide-induced rat tongue carcinogenesis. Oral Oncol 2004;40:910-5. 53. Vered M, Yarom N, Dayan D. 4NQO oral carcinogenesis: animal models, molecular markers and future expectations. Oral Oncol 2005;41:337-9.

54. Pommier Y, Jenkins J, Kohlhagen G, Leteurtre F. DNA recombinase activity of eukaryotic DNA topoisomerase I; effects of camptothecin and other inhibitors. Mutat Res 1995;337:135-45. 


\section{4-Nitroquinoline-1-Oxide Induces the Formation of Cellular Topoisomerase I-DNA Cleavage Complexes}

Ze-Hong Miao, V. Ashutosh Rao, Keli Agama, et al.

Cancer Res 2006;66:6540-6545.

Updated version Access the most recent version of this article at:

http://cancerres.aacrjournals.org/content/66/13/6540

Cited articles This article cites 54 articles, 21 of which you can access for free at:

http://cancerres.aacrjournals.org/content/66/13/6540.full.html\#ref-list-1

Citing articles This article has been cited by 5 HighWire-hosted articles. Access the articles at:

/content/66/13/6540.full.html\#related-urls

E-mail alerts Sign up to receive free email-alerts related to this article or journal.

Reprints and To order reprints of this article or to subscribe to the journal, contact the AACR Publications Subscriptions Department at pubs@aacr.org.

Permissions To request permission to re-use all or part of this article, contact the AACR Publications Department at permissions@aacr.org. 